\title{
Absolute Quantum Efficiencies
}

\author{
G. A. Crosby* \\ Department of Chemistry, Washington State University, Pullman, Washington 99163
}

J. N. Demas

Department of Chemistry, University of Virginia, Charlottesville, Virginia 22901

and

J. B. Callis

Department of Chemistry, University of Washington, Seattle, Washington 98105

(July 11, 1972)

\begin{abstract}
Recent developments in several areas of chemistry, laser technology, photodetector instrumentation, and calorimetry are surveyed, and their probable impact on the measurement of quantum yields is assessed. Chemical developments include: (a) synthesis and design of new luminescent molecules that could possibly serve as standards, (b) application of improved separation techniques to provide samples of extreme purity, and (c) advances in photochemistry that portend the development of widerange chemical actinometers. The potential use of lasers in quantum-yield measurements and their advantages over conventional sources for application in both optical and calorimetric techniques are pointed out. New methods of quantum-yield measurements, based on the novel characteristics of laser pump sources, are suggested, including the feasibility of measuring yields under time-resolved conditions and of employing internal standards. The possible lifting of wavelength restrictions on both laser sources and detector devices and the implications of these developments for extending the spectral range of quantum-yield measurements are discussed. The current status of calorimetry for determining yields is surveyed, and the impact of recent technology on the feasibility of developing calorimetric methods competitive with optical methods is assessed.
\end{abstract}

Key words: Calorimetry in quantum yields; laser, use in quantum yields; photodetectors in quantum yields; quantum efficiencies.

\section{Introduction}

The explosive developments of science and technology in the last quarter century have been mirrored in the steady rise of optical methods in chemistry, physics, and biology. Today the use of fluorescence and phosphorescence in fundamental and applied investigations is routine. Initially focussed on frequency and relative intensity measurements, the effort during the last few years has begun to encompass determinations of efficiencies of large classes of luminescent materials. Such a measurement, the quantum-yield determination, is one step removed in difficulty from recording energy, measuring relative luminescence intensity, or monitoring rate of decay (at least for times beyond a microsecond) of excited species. The result is that developments in quantum-yield technology have lagged behind those in the other areas. Recently, however, the technological advances relevant to quantum-yield measurements and the increased interest in obtaining efficiencies signal that a comprehensive analysis should be carried out on the present needs for accurate quantum efficiencies and an assessment be made of the current developments in the methods of determining them.

The present article is not an attempt to review the field of quantum-yield measurements. Although considerable space is devoted to optical and calorimetric methods for determining yields, no attempt has been made to review these techniques adequately either. (For a comprehensive review of optical methods, see ref. [1]. $\left.{ }^{1}\right)$ Rather the thrust of the present article is to point out a few of the obvious needs for accurate yield information, to note some of the current progress in 
instrumentation technology and chemistry that may lead to better yield determinations, and to suggest a few avenues of investigation that appear ready for development. Our remarks are restricted mainly to solutions and rigid glasses. For readers interested in yields from solid materials, we direct them to the review article by Lipsett and to the original literature $[2-10]$. Gas phase determinations are also not discussed, although recent publications show that the field is expanding [11-13].

\section{The Importance of the Quantum Efficiency}

The luminescence quantum efficiency of a compound $(\Phi)$ is defined as the fraction of molecules that emit a photon after direct excitation by the source. The terms absolute yield, quantum yield, yield, and efficiency are all used interchangeably throughout this article.

Recent research developments in many areas of physical, chemical, and biological research have reached a stage where the lack of absolute quantum efficiencies is seriously hampering further progress. We enumerate here a few areas where such information could be decisive. In theoretical chemistry, studies of radiative and especially radiationless processes have been proliferating [14-18]. The role of various terms within the Hamiltonian (vibronic, spin-orbit) [19-22] and the effect of substituents on both radiative and radiationless rate constants of isolated molecules are under scrutiny [23-25]. The interactions of excited species with the host molecules in condensed phases are not well understood, but some information is beginning to accumulate [26, 27]. Reliable efficiencies are needed on many classes of molecules to test the predictions implicit in the formalisms and to unearth new problems.

An especially important fundamental problem concerns the limits of applicability of the Strickler-Berg equation [28] for obtaining radiative lifetimes of excited states from the inverse absorption processes [29]. Serious discrepancies exist between the measured natural radiative lives $\left(\tau_{0_{m}}\right)$, as obtained from the equation $\tau_{0_{m}}=\tau_{f} / \phi_{f}$ where $\tau_{f}$ is the fluorescence decay time and $\phi_{f}$ is the fluorescence quantum yield, and those calculated by the Strickler-Berg relation. These discrepancies exist for simple molecules (e.g., $\mathrm{SO}_{2}$ ) [30] and for larger systems such as diphenylpolyenes [31]. The lifetimes can be anomalously long $[32,33]$ or anomalously short [34]. Large intermolecular [33] or intramolecular configurational changes [34] have been invoked to rationalize the results. More quantum efficiencies are required to quantify the experimental situation.

A second problem in the application of the StricklerBerg equation involves the selection of degeneracy factors for upper $\left(g_{u}\right)$ and lower $\left(g_{l}\right)$ states involved in the transitions when application is made to formally forbidden transitions $(S \rightarrow T)$. For hydrocarbons at the usual refrigerant temperatures $(\geqslant 50 \mathrm{~K})$ a value of $g_{u}=3$ is reasonable, since the level splitting is much smaller than $k T$ and thermal equilibrium is established among the nearly isoenergetic levels. For systems where spin-orbit coupling is large, such as encountered in $d-d$ [35] and charge-transfer [36, 37] excited states, the splittings mav be comparable to or even greater than $k T$. Then measured quantum yields and lifetimes are expected to be temperature dependent, and the Strickler-Berg relation can only really be applied and tested after careful studies of temperature-dependent decay times [38] and quantum yields [39] have been carried out. Reliable quantum efficiencies for many model systems are needed.

Another area where reliable quantum efficiencies are lacking, and one where the role of experimental yields can be decisive, is in determining the effects of deuteration on the radiationless rate constants for degrading excitation energy. Theoretical classifications of radiationless processes according to "strong" and "weak" coupling limits have been made. In the first case, no important deuterium effects on radiationless rates are predicted; in the latter, large changes due to deuteration are expected [40]. Few systems have been studied experimentally to distinguish these coupling limits. It is manifest that major decisions about the relative importance of various mechanisms for fundamental radiationless processes could be made from quantum-yield comparisons of deuterated and nondeuterated molecules [41-43].

The present status of organic photochemical research also warrants the effort of measuring accurate luminescence yields. Particularly important is a knowledge of efficiencies for organic molecules that can be used as energy donors or acceptors in photosensitization experiments. Reliable yields on series of closely related molecules whose singlet or triplet states span important energy regions would aid substantially in the design of experiments to test mechanisms of energy transfer and to control photochemical products. The need for yields of organic systems is compounded by the fact that mixed solvents and unconventional pure solvents are sometimes necessary. Thus, the known efficiencies of compounds must be extrapolated into systems where conditions differ radically from those prevailing during the original determinations.

Not only total quantum yields, as defined initially, but yields from particular levels in molecules exhibiting multiple level luminescence are needed. Such quantities are of paramount importance for assessing relative quenching rates of excited singlet and triplet levels, for obtaining efficiencies of intersystem crossing, and for relating measured quantities to theoretically defined parameters. The processes associated with a typical organic system are illustrated in figure 1. In terms of the rate constants displayed on the figure, we can define: $\phi_{f}=k_{f} /\left(k_{f}+k_{q_{f}}+k_{i s}\right), \phi_{p}=k_{p} /\left(k_{p}+k_{q_{p}}\right)$, and $\phi_{i s}=k_{i s} /\left(k_{i s}+k_{f}+k_{q_{f}}\right)$. It is obvious that $\Phi$, the total measured quantum yield as defined earlier, equals $\phi_{f}+\phi_{p}$ provided the only processes occurring after excitation are those depicted in the figure. It is also clear that the sum of $\phi_{f}$ and $\phi_{i s}$ may or may not be unity depending upon the existence of singlet quench- 


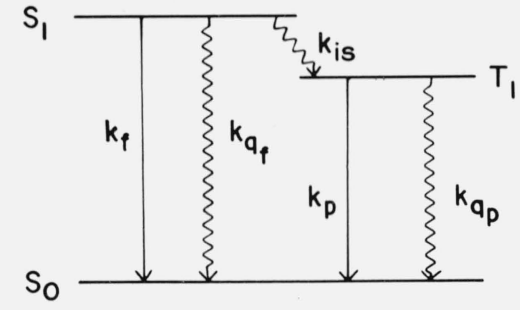

Figure 1. Principal pathways of degradation of an excited organic molecule. $\longrightarrow$, radiative decay; $\leftrightarrow$, radiationless decay. $k_{f}=$ radiative rate constant for depopulating $S_{1} ; k_{q_{f}}=$ radiationless rate constant for depopulating $S_{1} ; k_{i s}=$ radiationless rate constant for converting from $S_{1}$ to $T_{1} ; k_{p}=$ radiative rate constant for depopulating $T_{1} ; k_{q_{p}}=$ radiationless rate constant for depopulating $T_{1}$. $k_{f} /\left(k_{f}+k_{q_{f}}+k_{i s}\right)=\phi_{f}$ (quantum yield of fluorescence from $S_{1}$ ). $k_{p} /\left(k_{p}+k_{q_{p}}\right)=\phi_{p}$ (quantum yield of phosphorescence from $T_{1}$ ). $k_{i s} /\left(k_{i s}+k_{f}+k_{q_{f}}\right)=\phi_{i s}$ (quantum yield of intersystem crossing).

ing $\left(k_{q_{f}}\right)$. A particularly important quantity is $\phi_{i s}$. It measures the efficiency of populating the triplet state, thus producing a reactive species responsible for many photochemical reactions and frequently serving as an efficient energy donor.

Several methods have been proposed to determine $\phi_{\text {is }}$ [44-51]. Different photophysical properties anc processes have been utilized and ingenious techniques have been devised to extract $\phi_{i s}$ from the data. None theless, the methods rely eventually on the measure ment of a photoluminescence quantum yield. A funda mental question raised by these measurements of intersystem crossing yields is whether the sum of $\phi_{f}+\phi_{i s}$ is indeed unity. The equality has been shown to hold for a number of aromatics [52-57] and for porphin and its derivatives [58]. Exceptions abound, however, [59] and the experimental situation is still unclear [60]. Accurate and extensive quantum-yield measurements are necessary in order to establish or refute the stated equality as a fundamental (or rarely violated) law of luminescence. For pyrene, $k_{f_{q}}$ is reported to be negligible at $77 \mathrm{~K}$ but competitive with $k_{f}$ at room temperature [61].

The role of quantum-yield measurements in providing information on biologically important systems is expanding rapidly. Reports of quantum yields of naturally occurring bases [62] as functions of $\mathrm{pH}$ are providing insight into the possible fundamental physiological functions of these materials. Useful information on conformations and the nature of the excited states of biopolymers [63-65] has been provided from fluorescence measurements on oligonucleotides and polynucleotides containing fluorescent purine analogs. Some of the reported quantum yields are extremely low dictating new demands for accurate quantitative measurements at low light levels.

The real power and usefulness of fluorescent probes in biological systems are becoming appreciated. A typical example is 2-p-toluidinyl-6-naphthalenesulfonate (TNS), one of a series of compounds that are practically nonfluorescent in water but strongly fluorescent in organic solvents or when bound to protein molecules. This substance can be used as an extrinsic fluorescent chromophore for the study of protein conformations $[66,67]$, in the study of nitrogen antibody interactions [68], in following protein conformational changes produced by metal ion binding [69], and in elucidating enzyme activation processes [70]. In spite of its obvious importance it is significant that the mechanism by which the fluorescence of this molecule is changed by the medium is still not well understood. Spectroscopic studies, including detailed and accurate quantum-yield determinations, are of fundamental importance for molecules of this type and should be actively sought.

The need for quantum yields of inorganic molecules is also manifest and growing. Especially those materials classed as inorganic coordination compounds, usually containing some organic moieties, deserve considerable attention. This class of molecules, long utilized empirically in fluorescence metal analysis, has only relatively recently been recognized as providing candidates for electro-optical devices [71], for fundamental studies of radiative and radiationless transitions $[35,36]$, and for energy donors in inorganic photochemical studies [72]. The real possibility of engineering these molecules to have predetermined frequency ranges, high yields, and appropriate chemical properties [73] opens up a whole range of new experiments that were not possible just a few years ago. Needed, however, is detailed information on their spectroscopic properties, especially their absolute efficiencies, and the temperature dependences thereof. The near infrared region, virtually inaccessible at present to quantum-efficiency measurements can possibly be made accessible by the use of complexes possessing lowlying emitting levels, particularly those molecules displaying charge-transfer luminescence. Yet, few yields of these compounds have been measured, and none, to our knowledge, of compounds emitting beyond $800 \mathrm{~nm}$.

We digress here to present some information on a few representative transition-metal complexes whose unique luminescence properties are not yet well recognized. Complexes of ruthenium(II), osmium(II), and iridium(III) with $\pi$-conjugated ligands display visible and near-UV absorption spectra dominated by strong charge-transfer absorption bands of high intensity ( $2000 \leqslant \epsilon \leqslant 20,000$, where $\epsilon$ is the molar absorptivity) (figs. $2,3,4)$. In the case of osmium(II) complexes, the absorption bands extend well into the red region of the spectrum. Upon irradiation these molecules emit light with good efficiencies at low temperature $(>10 \%)$, and some are still reasonably efficient at room temperature in fluid solution. In figures 2 and 3 it is seen that the quantum yields are independent of wavelength, a property of fundamental and applied importance.

Other spectroscopic properties of interest of these molecules are evident in figure 4 . One sees that each emission spectrum consists of a unique electronic band. This is a feature characteristic of all these systems at 


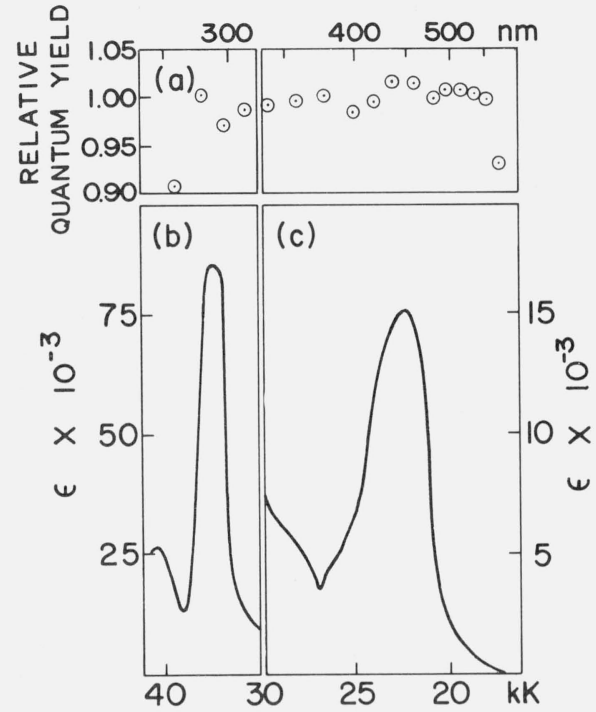

FIGURE 2. Relative quantum yield (a) and absorption spectrum (b, c) of tris $\left(2,2^{\prime}\right.$-bipyridine)ruthenium(II) chloride in methanol at room temperature: (a) $0.2 \mathrm{~g} / 5 \mathrm{ml}$ in a $1-\mathrm{cm}$ cell, (b, c) $6.7 \times 10^{-5} \mathrm{M}$ in a $1-\mathrm{cm}$ cell. The first and last yield points are less accurate than the others. [Ref: J. N. Demas and G. A. Crosby, J. Amer. Chem. Soc. 93, 2841 (1971).]

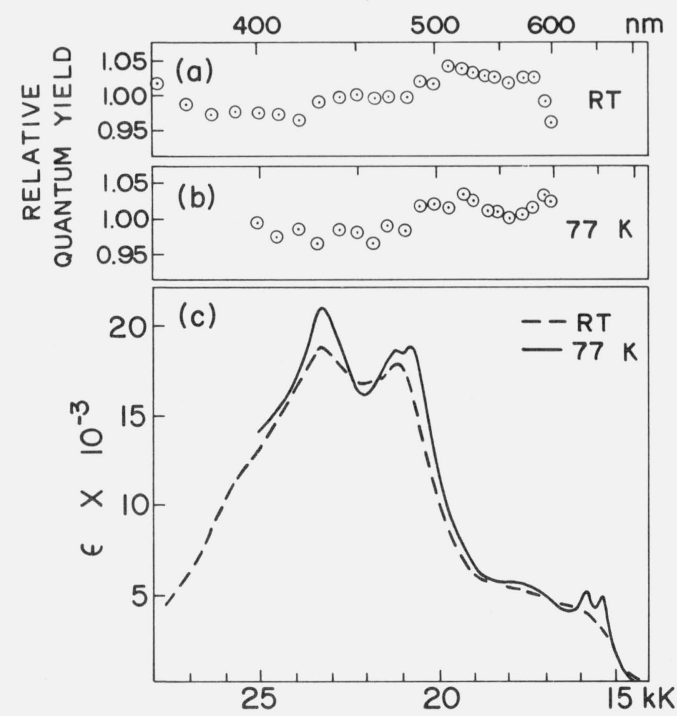

FIGURE 3. Relative quantum yields (a, b) and absorption spectra (c) or tris(1,10-phenanthroline)osmium(II) iodide in ethanol-methanol $(4: 1, v / v)$ : (a) $4.5 \times 10^{-7} M$ in a $1.76-\mathrm{cm}$ cell at room temperature; (b) $4.5 \times 10^{-7} M$ in a $1.76-\mathrm{cm}$ cell at $77 \mathrm{~K}$ (glass); (c) $\cdots, 9.0 \times 10^{-6} M$ in a $10-\mathrm{cm}$ cell at room temperature;,$- 7.12 \times 10^{-5} M$ and $1.42 \times 10^{-5} M$ in 1.76-cm cells at $77 \mathrm{~K}$ (glass). [Ref: J. N. Demas and G. A. Crosby, J. Amer. Chem. Soc. 93, 2841 (1971).]

temperatures above $77 \mathrm{~K}$ [35-37]. Furthermore, the measured decay times are on the order of microseconds, thus falling between those limits normally

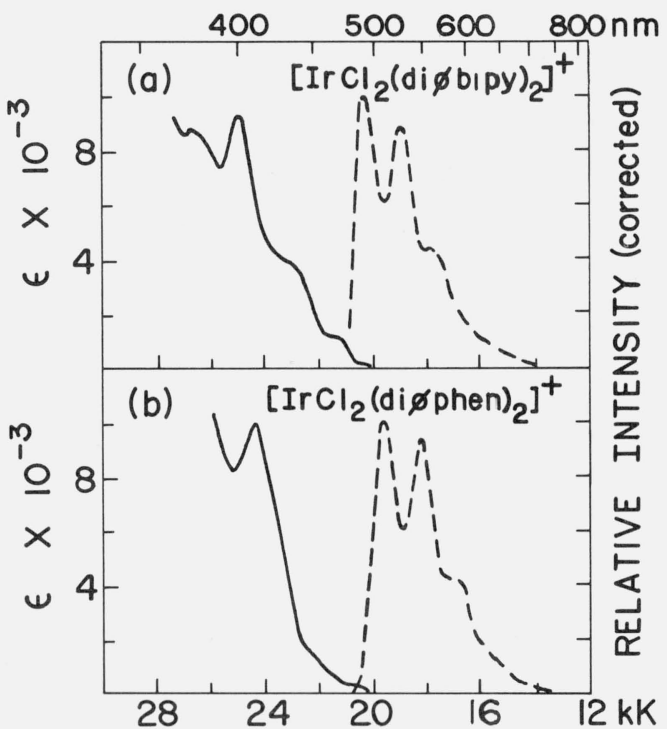

FIGURE 4. Absorption (- ${ }^{-}$) and luminescence (- - ) spectra of phenyl-substituted iridium(III) complexes in ethanol-methanol glass $(4: 1, v / v)$ at $77 \mathrm{~K}$. (a) cis-dichlorobis $\left(4,4^{\prime}\right.$-diphenyl-2, $2^{\prime}$-bipyridine)iridium(III) chloride, (b) cis-dichlorobis(4,7-diphenyl-1,10-phenanthroline)iridium(III) chloride. [Ref: R. J. Watts and G. A. Crosby, J. Amer. Chem. Soc. 94, 2606 (1972).]

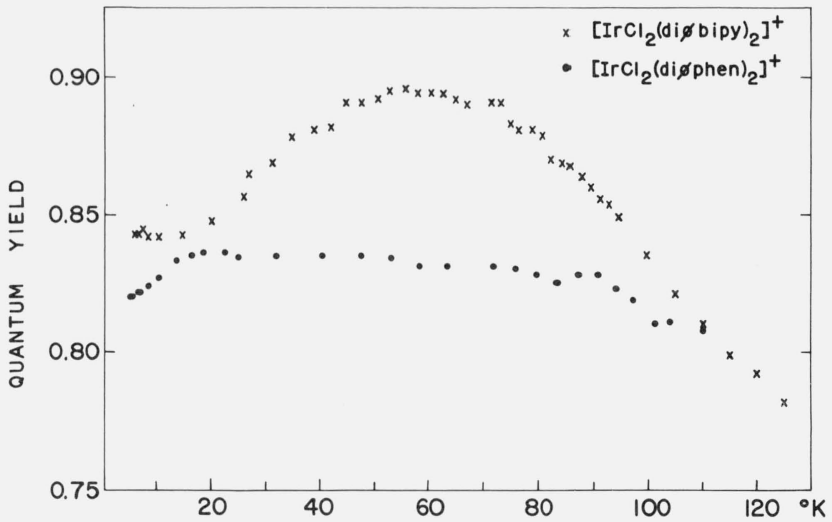

Figure 5. Temperature dependence of quantum yields. $\times$, cisdichlorobis(4,4' -diphenyl-2,2' -bipyridine)iridium(III) chloride. $\bigcirc$, cis-dichlorobis(4,7-diphenyl-1,10-phenanthroline)iridium(III) chloride. Both molecules were dissolved in poly(methylmethacrylate) and irradiated with $\sim 365 \mathrm{~nm}$ radiation.

encountered for established fluorescences and phosphoresences. Finally, the capability of tuning the frequency by ligand change is apparent.

Additional spectroscopic features of importance are evident in figure 5. Not only are the yields of some of the molecules extremely high but, also, for some of them, the efficiencies are virtually temperature independent over large ranges. Because of the favorable chemical properties of these substances and their unique luminescence behavior, considerable fundamental information concerning the excited state properties of transition-metal complexes can be gleaned 
from studies of their photoluminescences. Particularly important are accurate determinations of quantum yields as a function of temperature [38, 39]. Their possible value as fluorescence standards and as components in chemical actinometers is discussed below.

\section{Standards}

The absolute determination of quantum yields is a measurement fraught with difficulties. Understandably, workers have resorted to the much more convenient and rapid technique of measuring yields relatively, i.e., by comparison with known standards. Perforce, this procedure shifts a major burden of the accuracy of the final figure to the standard. Thus, the real need for more and better standards. Compounding the problem is the necessity to have standards for various solvents, for various temperature ranges, for wide ranges of wavelengths, and for different states of matter (solid, plastic, glasses, solutions). This is a stiff order. When one considers the importance of standards, the small number of well-studied ones, and the disconcerting disagreement of reliable workers on the actual reported efficiencies, the magnitude of the problem of obtaining good standards is revealed. [Relative yield measurements are so much easier to perform than absolute ones, however, that the demand for absolute standards will continue to increase.] An account of commonly used standards and an assessment of the reliability of their yield values are available [1]. Additional information on common standards and some interesting suggestions for new ones are contained in the article by Testa [74]. Many workers choose standards possessing favorable chemical properties for relative measurements even though the accuracy of some of the "standard" yields is not well substantiated.

The incidence on the market of commercial instruments that can measure yields routinely also increases the need for standards. In fact, institution of a program to remeasure old standards and search for new ones has considerable merit, in our opinion. If a bank of reliable standards could be built and marketed, which would provide checks on both research results and instrumentation throughout the country, a mass of misinformation would be clarified, and future additions of shoddy yield data to the literature would be measurably reduced. Such a program seems appropriate for the National Bureau of Standards.

At this juncture we wish to point out again the unusual optical properties of inorganic complexes, especially those of ruthenium, osmium, and iridium described above. It is our contention that they deserve serious study as candidates for standards, as well as for numerous other applications in spectroscopy and photochemistry. Because their properties can be modified by ligand substitution, ligand modification, and change of counter ion, it is possible to design them to have specified frequencies, high yields, and desirable solubilities. Thus, molecules can be engineered to dissolve in most common solvents. Their potential as quantum-yield standards should be studied, especially if internal standards (see below) were employed.

Finally, we wish to call attention to recent developments in purification procedures that bear on the accuracy of quantum-yield measurements. Chemical purity of any fluorescent compound is always desired; for quantum-yield standards it is a stringent requirement. This aspect of quantum-yield work has been repeatedly emphasized [1, 29, 75], not only for quantum-yield measurements but for emission spectroscopy in general. Intense light sources commonly employed to measure low yields exacerbate the impurity problems. The use of lasers dictates adherence to criteria for chemical purity far stricter than those normally applied [76]. Increases in detector sensitivity and the employment of high aperature equipment to monitor weak emitters place still greater demand for high standards of chemical purity. The availability of image intensifier tubes adds a new dimension to the impurity problem [77].

The virtues of thin-layer chromatography, column chromatography, and zone refining as supplements to the classical chemical techniques for obtaining high purity are relatively well known. Not commonly appreciated, however, are the remarkable chemical separations obtainable with the use of dextran molecular sieves in column chromatography. Although usually employed for biochemical separations, the effectiveness of molecular sieves for purifying inorganic materials is not common knowledge [78]. Indeed, the usual conception of the primary mechanism responsible for such separations (dominant role of molecular size) leads one to predict that molecules of similar size and shape should not be separable with such procedures. Yet, separations of isomeric, six-coordinated complexes of transition metals have been accomplished [73]. In fact, the discriminatory ability of the Sephadex gels to separate the components of reaction mixtures is so acute that surface adsorption activity must be at work in addition to steric effects [79].

Because of the many desirable features of gel filtration chromatography as a purification technique we strongly recommend its use both for purifying fluorescence standards and for preparing samples in general. For transition-metal complexes where reaction mixtures can produce a host of chemically and spectroscopically similar molecules, efficient separations are mandatory. In many cases gel filtration provides a superb answer to the problem.

\section{Quantum Efficiencies by Optical Methods}

The first reliable method for measuring quantum yields by an optical technique was introduced by Vavilov in 1924 [80]. The method is a substitution technique employing a solid scatterer as a standard. The experiment requires two basic measurements. First, the detector monitors the sample luminescence that is generated by total absorption of the excitation light focussed to a point in the cell. Then the detector 
records the light diffusely scattered by a magnesium oxide surface substituted for the original cuvette. From these data and some ancillary measurements, the absolute quantum yield can be computed. A detailed description of the technique including the derivation of equations is given in reference 1 . Basic improvements on the method were the introduction of a quantum counter by Bowen in 1937 [81] and the incorporation of the refractive index correction by Förster in 1950 [82]. Refinements by McClure et al. [83] in the early 50's and the introduction of the rhodamine $B$ quantum counter in 1955 by Melhuish [84] resulted in a method of measurement that has produced some of the most accurate quantum yields available.

In 1957 Weber and Teale [49] made a step forward by the introduction of solution scatterers for standards. This technique introduces its own special problems and limitations but is easier to carry out than the basic Vavilov method [1]. Numerous workers have devised improvements and modifications of both the Vavilov and the Weber-Teale methods for determining quantum yields, yet the measurements are still relatively tedious to perform and have many associated problems.

The difficulties inherent in absolute procedures and the demand for yield measurements on hosts of compounds have led workers to devise simple relative methods for performing the measurement. In these methods the yield of an unknown is compared with the "known" quantum yield of a standard material. Relative methods fall into two categories, optically dense and optically dilute. In the former, concentrated solutions are employed to absorb all the incident flux; in the latter, dilute solutions insure negligible diminution of the excitation beam across that part of the sample viewed by the detector. Optically dense quantum-yield measurements relative to a standard can be made extremely rapidly and are subject to moderate errors provided the substances satisfy somewhat rigid requirements. The accuracy can be low due to effects associated primarily with high concentrations. Optically dilute methods generally avoid problems created by solute-solute interactions and, due to the availability of high quality spectrofluorometers and their components, have become the preferred techniques for obtaining yields.

An analysis of optically dilute methods, the useful equations and their limits of validity, and the problems associated with this kind of measurement are contained in reference 1 . We refer the interested reader to this review and the references therein. We wish to point out here, however, two problems associated specifically with yields obtained by optically dilute procedures that modern laser technology should admirably solve, (a) inaccurate (high) yields caused by strong light from a broad band source leaking through the filter system and (b) inaccurate yields produced by uncertainties in the effective absorbances of samples over the finite bandpass of the filters or monochromator excitation system (see Lasers, below).

For routine determinations of quantum yields by optical methods, the use of optically thin solutions versus optically thin standards is probably the best method available. The reported reproducibility is quite good (1-2\%), and the agreement between laboratories is excellent $(<5 \%)$ for some molecules. Considerable improvement in accuracy is to be expected from substituting lasers for conventional sources and from incorporating modern electronics and detectors into the systems. Although frequently reported with high precision [35-37], the accuracy of quantum yields obtained from substances dissolved in rigid glasses may not be high in spite of the good reproducibility. The relative importance of polarization errors is especially vexing to assess for rigid systems, and more work on this aspect of the optically dilute methods is badly needed [85].

Finally, we reiterate that both the optically dense and optically dilute techniques are still relative ones. Whatever systematic errors are built into the standards will be reflected in the results. Accurately measured standards are urgently needed.

\section{A. Recent Developments in Instrumentation}

We turn now to highlight some recent instrumentation advances that bear on optical methods of determining quantum yields. Lasers are by far the most important development, but the significance of available high sensitivity Raman instrumentation and recent improvements in photodetectors deserve attention.

\section{Lasers}

Lasers are now available in a wealth of types, powers, and configurations. The salient features of the most common varieties are reviewed in table 1 . This list is by no means complete. In many cases, commercially available items are listed, and data have been drawn frequently from the manufacturer's literature. It should be further noted that specialized design frequently can improve many of the listed characteristics. In addition, because of the rapid advances in laser technology, this table will surely be obsolete by the time it is printed. The interested reader should consult the latest issues of the laser trade journals (e.g., Laser Focus and Laser Sphere) and other technical journals for up-to-date information. An especially valuable source is reports on advanced seminars in laser technology [86].

Most significant for luminescence and quantumyield work is the high continuous wave (cw) powers available in the near UV and visible from argon lasers. The continuous tunability of the cw pumped dye lasers and their remarkably narrow line widths are also particularly noteworthy. Pulsed nitrogen lasers with their extremely short duration pulses and UV frequencies are ideally suited for most lifetime measurements and even for nanosecond photolysis. By driving a dye laser with a nitrogen laser and selecting the wavelength with an intracavity grating, one can preserve the extremely short durations and relatively high powers (one to two orders of magnitude in power are sometimes lost), but the added feature of continuous tunability from the 
TYPE

Discrete Line Lasers

Ar ${ }^{a}$

$\mathrm{Kr}^{\mathrm{a}}$

\section{Tunable Lasers}

cw pumped dyes with intra-

cavity $\lambda$ selection ${ }^{a}$ $\lambda, \mathrm{nm}$

$351.1+363.8 ; 457.9 ; 472.7 ; 476.5$; $488.0 ; 496.5 ; 501.7 ; 514.5 ; 528.7$

$350.7+356.4 ; 476.2 ; 482.5 ; 520.8 ;$ 530.9 ; 568.2; 647.1; 676.4; 752.5; 799.3

520-690 $10-160 \mathrm{~mW}$
MONOCHROMATICITY

$20 \mathrm{~mW}$ to $2 \mathrm{~W}$

$<0.001 \AA$

$20-500 \mathrm{~mW}$

$<0.001 \AA$

$0.1 \AA$ normal to $0.001 \AA$

\section{Pulsed Lasers}

TYPE

Discrete Line Lasers

$\mathrm{N}_{2}{ }^{\mathrm{b}}$

$\mathrm{Nd}^{3+}$.YAG

Ruby
$360->650$

$\mathrm{N}_{2}$-laser pumped dyes ${ }^{\mathrm{b}}$

$\mathrm{Nd}^{3+}$-YAG pumped dyes ${ }^{\mathrm{e}}$

Parametric oscillator ${ }^{\mathrm{e}}$ $\lambda, \mathrm{nm}$

337.1

266 (frequency quadrupled), 13 lines from 473 to 679 (frequency doubled), 13 lines from 946 to 1358

694 (Q-switched)

347 (frequency doubled)
POWERS

$1-10$

$1-100 \mathrm{~kW}$ peak

$0.5 \mathrm{~W}$ average

$1-5 \mathrm{~kW}$ peak

10-100 $\mathrm{m} \mathrm{W}$ average
MONOCHROMATICITY

$\sim 1 \AA$

$<1 \AA$

$100-400$

$100 \mathrm{MW}$ peak

$1 \mathrm{~J}$ per pulse

$10 \mathrm{MW}$ peak

$0.25 \mathrm{~J} /$ pulse

$600 \mathrm{~kW}$ peak

$25 \mathrm{~mW}$ average

2-150 $\mu \mathrm{J} /$ pulse

$1-10 \mathrm{~kW}$ peak

1-50 $\mathrm{m} \mathrm{W}$ average

2C. $700 \mathrm{~W}$ peak

$0.2-2 j \mathrm{~mW}$ average

50-700 W peak 1-10 $\mathrm{mW}$ average
$0.1 \AA$

$20^{d}$

$0.1 \AA$

$20^{d}$

$<1-10 \AA$

$100-500^{\circ}$

$<1-20 \AA$

$2-10^{d}$

$<1 \AA$

$\leqslant 1 \AA$ to $0.001 \AA$

70-150
DURATION nsec

5-10

Tunable Lasers

\footnotetext{
${ }^{a}$ Data for lasers from Spectra-Physics and Coherent Radiation.

${ }^{\mathrm{b}}$ Data for AVCO Everett Research Lab. lasers.

${ }^{\mathrm{c}}$ Data for Synergetics Research, Inc. lasers and from references
}

${ }^{d}$ Mode locking can yield picosecond pulses ( $<0.1$ nsec), although spectra bandwidth will usually be increased. See references $[90,91]$.

${ }^{\mathrm{e}}$ Data for Chromatix laser. 
near UV through the visible is obtained. These characteristics are ideal for carrying out time-resolved spectroscopy, a powerful fundamental and analytical tool. The large pulse energies available from the flashlamp-pumped dye lasers are also attractive for some studies, and systems can be built for minimal cost [87-89].

From table 1 the benefits accrued from replacing conventional light sources with a laser source are manifest. The powers available far exceed those normally obtained from even wide bandpass conventional sources. This power gain, however, can be obtained at no loss in spectral purity, and for most molecular systems one can treat the sample absorbance as constant over the excitation line. Thus, one of the significant sources of error mentioned above in quantum-yield and excitation-spectra determinations is eliminated. The higher powers available from the lasers also permit the use of more dilute solutions; this reduces the trivial errors of self absorption and secondary emission.

In addition to the high powers and narrow line widths, lasers have other advantages. Typically, the laser beam is of very small size. A cw argon laser, for example, has a beam diameter of 1 to $2 \mathrm{~mm}$. It is highly coherent and can be focussed to an essentially diffraction limited spot. This high degree of focusability is commonly exploited in Raman work where the laser is directed down the long axis of a microcapillary. The use of a microcapillary cell would also yield significant advantages in luminescence spectroscopy. There would be negligible reabsorption, and the source composed of the emitting volume would be a near line. Thus the emissions could be focussed extremely efficiently onto the entrance slit of an emission monochromator, even for very narrow emission bandpasses. Such a system could not be used with even moderately photosensitive materials, however, unless a flow system were employed. Microcapillary cells have a further disadvantage for comparative work due to the higher precision required for reproducible alignment.

There seems little doubt that the wavelength range, the pulse duration, and the available powers of most of the types of lasers will be greatly improved. With progress in mode-locking [90-92] it seems reasonable to anticipate that the dye lasers will yield repetitive, stable, and continuously tunable subnanosecond pulses extending from the UV to near-IR regions. Such a tunable, short duration source would represent truly a delta function excitation flash for luminescence lifetime measurements and time-resolved spectroscopy, with little sacrifice in power or spectral purity. For dye lasers especially, considerable improvements in peak and average power are anticipated as the pulsed nitrogen lasers become more efficient. Wavelength range limitations of tunable dye lasers will almost certainly become relaxed as the dyes are frequency doubled into the UV, and new dyes will surely extend the available range well into the infrared. Already the entire range between 7000 and 11,700 $\AA$ has been covered by pump- ing photographic film sensitizer dyes with Q-switched ruby lasers [93]. A number of parametric oscillators has been constructed that exhibit continuous tunability from 7000 to $20,000 \AA$, and efficiencies of 45 percent have been reported [94].

Referring to the optical quantum-yield methods sketched above, we cannot think of a technique that would not benefit substantially from the replacement of the conventional light source with the laser. We have no doubt that where low cost is not a prime factor, but accuracy is of paramount importance, lasers will eventually become the excitation source of choice. This statement applies to calorimetric methods also.

\section{Raman Instrumentation}

The developments of laser Raman spectroscopy have yielded a whole new line of optical components that are admirably suited for luminescence and quantumyield measurements as well. One of the principal areas of progress is monochromator technology. Quite fast, extremely-low stray-light, double monochromators have been produced. Stray light in these devices is so low that one can scan to within a few wave numbers of the laser line pump. Such superb stray light rejection makes them a natural choice for luminescence and quantum-yield measurements, especially when laser excitation is employed. It is noteworthy that much of the impetus for the development of very high power krypton and argon lasers also stemmed from the needs of the Raman spectroscopist.

Photomultipliers have also undergone enormous improvements. By reducing the effective photocathode size and cooling the tubes to reduce the dark current, the dark backgrounds have been reduced to a few counts per second (e.g., tubes of the IT\& $\mathrm{T}^{2}$ type). All this has been accomplished with an extended red response S-20 photocathode, which may exhibit a quantum efficiency of several percent beyond $800 \mathrm{~nm}$.

Thus the modern unmodified Raman instrument using an argon laser source represents a nearly ideal excitation-detection system for luminescence, at least when the molecules absorb in the region of the laser lines. The ready availability of these Raman spectrometers within many laboratories also adds to their attractiveness for such work. Almost any further advances in Raman technology are certain to be adaptable with benefit for making luminescence measurements and for determining quantum efficiencies.

\section{Photodetectors}

In addition to the restricted area tubes used in Raman spectroscopy, there have been other signifcant advances in detector technology. RCA, for example, has introduced a line of gallium arsenide and indium-doped gallium arsenide extended red-response

\footnotetext{
${ }^{2}$ In order to adequately describe materials and experimental procedures, it was occasion ally necessary to identify commercial products by manufacturer's name or label. In no instances does such identification imply endorsement by the National Bureau of Standards, nor does it imply that the particular product or equipment is necessarily the best available
} for that purpose. 
photomultipliers. The gallium arsenide tubes typically have quantum efficiencies of 1.4 percent at $860 \mathrm{~nm}$. With indium doping, the red response is further extended, and RCA will supply tubes having efficiencies of 1 percent at $950 \mathrm{~nm}, 0.2$ percent at $1000 \mathrm{~nm}$, and 0.1 percent at $1060 \mathrm{~nm}$. In addition to having remarkably deep infrared penetration, these tubes have quite low dark currents even at room temperature; in most cases they can be regarded essentially as a quantum jump better than the old infrared tubes with $\mathrm{S}-1$ response. Further improvements seem likely since gallium arsenide photosensitive surfaces have been prepared having maximum quantum efficiencies of 80 percent [95].

Semiconductor diodes are also improving. Germanium diodes of the type used in nuclear instrumentation make superb optical detectors [96]. They can have large areas $(>1 \mathrm{~cm} \times>1 \mathrm{~cm})$ and sensitivities comparable to photomultipliers. Their noise equivalent power (NEP) is $\sim 10^{-15} \mathrm{~W} / \mathrm{Hz}^{1 / 2}$ from the visible to beyond $1600 \mathrm{~nm}$. Their stability and near quantum flat response of $\sim 0.6$ has led to the suggestion that they be used as replacements for thermopile radiation detectors [96].

When operated in the avalanche mode, these photodiodes have many of the characteristics of photomultipliers. They still have the high quantum efficiencies of the solid state photodiodes but also exhibit a limited gain multiplication. Typically, their sensitivities stretch well into the near infrared. Texas Instruments, for example, has introduced a series of silicon and germanium photodiodes packaged with amplifiers (TIXL 74-76). For the silicon system, the NEP at 900 $\mathrm{nm}$ is less than $5 \times 10^{-13} \mathrm{~W} / \mathrm{Hz}^{1 / 2}$. For the germanium ones, the NEP is $\sim 2 \times 10^{-12} \mathrm{~W} / \mathrm{Hz}^{1 / 2}$ at $1060 \mathrm{~nm}$ or $\sim 6 \times 10^{-12} \mathrm{~W} / \mathrm{Hz}^{1 / 2}$ at $1540 \mathrm{~nm}$. With properly biased photodiodes, single photon counting is even possible. With germanium photodiodes, NEP's of $\sim 10^{-13}$ $\mathrm{W} / \mathrm{Hz}^{1 / 2}$ at $1500 \mathrm{~nm}$ have been realized. Germanium devices are usable to beyond $1700 \mathrm{~nm}$ [97]. It seems reasonable that further improvement in these avalanche devices will yield even higher sensitivity in the nearIR region. It is even possible that they will become competitive with photomultipliers in the visible-UV regions. In a spectrophotometry application weak transient absorption during flash photolysis has been detected in the $0.6-1.1 \mu$ region with the aid of an inexpensive silicon photodiode [98].

If the major source of noise in an optical experiment is due to the photon statistics, then the signalto-noise ratio depends upon the square root of the quantum efficiency of the detector. Thus for high light level experiments the high quantum efficiency of photodiodes is a distinct advantage for noise discrimination, especially in the near infrared.

\section{B. New Experimental Techniques}

\section{Internal Standardization}

Internal standardization is frequently used in analytical chemistry. To date, however, we are aware of no use of an internal standard for quantum-yield measurements. This failure has been a result of the experimental difficulties involved rather than because of any undesirable fundamental features of the technique itself. In fact, internal standardization is probably one of the more elegant and useful techniques for accurate relative quantum-yield determinations. The method appears especially feasible in view of modern instrumental developments.

In the internal standardization procedure both the unknown and standard would be contained in the same solution. The emission spectra of the two compounds would then be run in a single scan of the emission instrument. From the relative emission intensities of the standard and the unknown and their absorbances one would then calculate the efficiency of the unknown relative to the standard by normal procedures. The advantages of such a procedure are numerous. Since the standard and unknown are in the same solvent and cell, refractive index corrections are all but nonexistent, and radiation trapping is essentially identical between both samples. Errors introduced by cell mismatch are eliminated. There are, of course, serious restrictions on the method. The standard and the unknown must emit in well-resolved spectral regions (by means of time-resolved spectroscopy, even this restriction may be lifted). They must also absorb at the same wavelength.

Several experimental difficulties must be considered closely if satisfactory results are to be obtained using an internal standard. Because the standard and unknown are present in the same solution, great care must be taken to prevent any bimolecular quenching or energy-transfer processes, since their occurrence would modify the yields of the species present [99]. As long as one is using optically dilute solutions, there is no problem with long-range energy transfer of the Förster-type. This would not, however, always be true if optically dense solutions were employed.

Diffusion-controlled processes must also be considered. One can readily show that even if a sample has a component with a lifetime as long as $1 \mu$ s that is quenched by the other one at the diffusion controlled limit $\left(10^{10} M^{-1} \mathrm{~s}^{-1}\right)$ then the concentration of the second species must exceed $10^{-6} M$ to cause 1 percent quenching of the first. Since optically dilute measurements are usually carried out at optical densities less than 0.01 , then any second component with $\epsilon \geqslant 10^{4}$ at the exciting wavelength would introduce a negligible error. Of course, if the first compound has a lifetime of less than $1 \mu$ s or the quenching proceeded with a rate less than the diffusion-controlled limit, then this already weak restriction would be relaxed still further. For example, if the standard's lifetime were $10^{-8} \mathrm{~s}$ (a typical fluorescence lifetime), then the maximum permissible concentration of the second species would be $10^{-4} \mathrm{M}$; this would permit molecules with $\epsilon \geqslant 10^{2}$ to be measured. The same arguments apply to the second component. Most materials and standards would have coefficients satisfying this condition in some region. Thus, because of bimolecular 
quenching it appears that the internal standardization technique is largely limited to optically dilute measurements. Consequently, all of the restrictions and limitations on any optically dilute method are present [1].

The remaining serious difficulty or source of error envisioned in a procedure employing an internal standard is that inherent in accurately calibrating any spectrometer over a wide wavelength range. Also, some compounds have extremely wide emission bandwidths; then difficulty will be encountered in obtaining satisfactory standards. Transition-metal complexes, as discussed above, may possibly be designed for appropriate standards. Sensitivity, even when working with low yield substances under optically dilute conditions, does not appear to present a stvere limitation for the method.

In summary, we believe that the use of an internal standard in relative quantum-yield determinations deserves serious consideration for investigation and development. If well-defined standards become readily available, the method could eventually become one of the preferred ways of running high-accuracy yield determinations.

\section{Quantum Efficiencies by Actinometric Methods}

The principle of the actinometric method for measuring quantum yields is conceptually simple. One merely surrounds the sample completely with an actinometer solution, measures the apparent intensity of the emitted radiation, and then, using the same type of actinometer solution, monitors the intensity of the excitation beam. If the irradiated solution is totally absorbing, the ratio of the two intensities is the absolute quantum yield. Whereas actinometers are capable of measuring absolute intensities, this technique is, in fact, a relative method since the absolute yield of the actinometer need not be known. To our knowledge, this method has not been used for measuring optically excited luminescence efficiencies. It has, however, been utilized in a suitably modified form for measuring chemiluminescence yields [100].

The principal reason for the nonadoption of an actinometric method is experimental. Most actinometers have quantum yields that are strongly wavelength dependent, and their absorption characteristics make it difficult to arrange to have all of the emitted light absorbed over the very wide wavelength ranges encountered in molecular luminescences. Because of these factors it is difficult to obtain an accurate measurement of the intensities of both the excitation beam and the broad molecular emission with the same actinometer. With the advent of new sources and actinometers, however, actinometric methods may achieve a useful place in absolute quantum-yield measurements.

before launching into speculative discussion we point out that the potassium salt of trans$\left[\mathrm{Cr}\left(\mathrm{NH}_{3}\right)_{2}(\mathrm{NCS})_{4}\right]$ (Reinecke) is an excellent widerange actinometer covering the 300 to $600-\mathrm{nm}$ region
[101]. Its yield has been measured over an extended wavelength range, and it is reasonably sensitive. Unfortunately, it is not without its shortcomings. To absorb most of the emitted light in the 300 to $600-\mathrm{nm}$ range, rather concentrated and/or thick solutions are required (about $1.5 \mathrm{~cm}$ at a concentration of $5 \times 10^{-2}$ $M)$. The compound also exhibits a slow but bothersome thermal decomposition. Finally, there is a slight, although perhaps not real, variation of the quantum yield with wavelength, a variation that is, however, small enough that reliable corrections could be made. No one has confirmed the original yields, and the initial data were taken at somewhat greater wavelength intervals than would be desirable for precise corrections. Notwithstanding these objections, Reinecke's salt is even now usable as a wide-range actinometer for yield determinations.

Recent photochemical investigations portend the development of additional wide-range actinometers. Demas and Adamson [102] have found that tris $\left(2,2^{\prime}\right.$. bipyridine)ruthenium(II) sensitizes, by a diffusioncontrolled process, the redox decomposition of tris(oxalato)cobaltate(111). Since the donor has a broad, intense absorption, it is quite easy to obtain, with thin solutions, total absorption of incident light from the UV to beyond $500 \mathrm{~nm}$. The decomposition of the $[\mathrm{Co}-$ $\left.\left(\mathrm{C}_{2} \mathrm{O}_{4}\right)_{3}\right]^{3-}$ may be readily monitored spectrophotometrically (unfortunately not with quite the high sensitivity of the ferrioxalate). Furthermore, since the efficiency of population of the donor level is believed to be unity and to occur virtually instantaneously following excitation, the system has a decomposition yield that is inherently wavelength independent and that has a broader range than most other actinometers. In its present form, their system is not, however, a useful actinometer. With modifications it, or a related system, may become practical.

Lasers make the actinometric method for measuring yields appear even more attractive. The excellent focusability of lasers permits an extremely small entrance aperture to be used and thus minimizes the error due to loss of radiation through the excitation port. Also, because the laser beam is inherently well collimated, one could use relatively dilute solutions in very long cells and irradiate down the cylindrical axis. In this way trivial reabsorption effects would be minimized. A device built along these lines might look much like a condenser with the outer cooling jacket filled with the actinometer solution. Of course, any measurement based on an optically dilute method would be subject to the normal corrections, and the optically dense method would be prone to its usual inherent errors. In spite of these objections, common to all optical quantum-yield measurements, it seems that the actinometric method has distinct possibilities even now with potassium Reinecke, and it will probably become more attractive with time, especially as the developments in modern inorganic photochemistry gain momentum and uncover new photochemical reactions and sensitizers. 


\section{Quantum Efficiencies by Calorimetric Methods}

Although measurement of quantum efficiencies optically involves generally familiar instrumentation, this statement cannot be made about calorimetric procedures. Recently, however, developments in technology point toward a gaining importance of calorimetry in the field of quantum yields, and we wish to discuss some of them. Because of their relative unfamiliarity, we present here a more detailed account of the various methods employed in calorimetry than was included above for the optical methods.

Calorimetric methods of measuring quantum yields are based on the assumption that energy absorbed from a beam of light incident upon a solution of fluorescent molecules is either lost through reemitted photons or degraded to heat by radiationless processes. The objective of calorimetric techniques is to measure the energy yield of these radiationless processes, $Y_{h}$, which is defined as the ratio of heat energy produced to incident photon energy absorbed. $Y_{h}$ is obtained by measuring the ratio of the heating produced by irradiation of the fluorescent sample to the heating produced by irradiation of a nonfluorescent sample that absorbs the same fraction of the excitation energy.

In the absence of photochemistry, the fluorescence [103] energy yield is the complement of $Y_{h}$ and the quantum yield of fluorescence is related to it by the formula

$$
\Phi=\left(\bar{\nu}_{a} / \bar{\nu}_{f}\right)\left(1-Y_{h}\right)
$$

where $\bar{\nu}_{a}$ and $\bar{\nu}_{f}$ are the average frequencies $\left(\mathrm{cm}^{-1}\right)$ of absorbed and fluorescence photons, respectively, defined by the formulas

$$
\begin{gathered}
\bar{\nu}_{a}=\left[\int I_{a}(\lambda) A(\lambda) d \lambda / \lambda\right] /\left[\int I_{a}(\lambda) A(\lambda) d \lambda\right] \\
\bar{\nu}_{f}=\left[\int I_{f}(\lambda) d \lambda / \lambda\right] /\left[\int I_{f}(\lambda) d \lambda\right]
\end{gathered}
$$

$I_{f}(\lambda)$ and $I_{a}(\lambda)$ are the fluorescence and excitation intensities at wavelength $\lambda, A(\lambda)$ is the percent absorbance of the sample with a pathlength equal to the cell dimension, and the integrals are over the fluorescence and excitation bands, respectively. Since $\bar{\nu}_{f}$ and $\bar{\nu}_{a}$ are easily measured with high precision by standard photometric techniques, they will not be discussed further here [1,29].

Several important factors favor calorimetric methods for the determination of absolute quantum yields: (a) The method serves as a valuable independent check of the assumptions used in photometric techniques for measuring $\Phi_{f}$; in particular, corrections for experimental geometry, index of refraction changes, and polarization effects are largely eliminated. (b) Using relatively crude apparatus, some workers have obtained values of $Y_{h}$ with a precision comparable to those obtained by other methods [29]. (c) The calorimetric method works well with samples that emit in the near infrared where the sensitivity of optical techniques is relatively low. Recent developments in photodetectors are alleviating this problem, however (see above).

With the usual procedures and components, the principal disadvantages and limitations of the method have been the following: (a) The insensitivities of the calorimeters used thus far have forced workers to use highly absorbing samples; consequently, large corrections for reabsorption of the fluorescence [29, 104] are required. (b) The method has been limited to substances possessing high quantum yields. (c) Measurements have been confined to liquid samples at room temperature. (d) Methods devised thus far have been tedious and time consuming to perform.

Recent advances in calorimetry, especially in the technology of light sources and transducers, and improvements in methods of data acquisition portend wider acceptance of the technique for obtaining quantum efficiencies. The generality, sensitivity, and ease of performance of calorimetric methods will undoubtedly improve. Our purpose here is to review some of the advances in methods and components and to indicate possibilities for still greater improvements.

\section{A. Calorimeters}

The calorimetric apparatus consists of an excitation source, a means for selecting a bandwidth of the incident radiation, and a calorimeter to measure the heat energy produced during irradiation. The science of calorimetry is a mature field of endeavor, and the reader desirous of a general background in the subject is referred to the several books and recent reviews now available [105-108]. Let us begin ou discussion of calorimetric apparatus by considering the constraints on the calorimeter imposed by the requirements for measuring quantum yields. First, provision must be made for admitting the exciting light. Second, the calorimeter must be designed so that all fluorescence escapes the cell. Third, since the sample will not be uniformly irradiated, the average or total heating must be detected. Fourth, the calorimeter must be very sensitive. In general, the sensitivity requirements will depend on the energy of the incident radiation, the absorbance of the sample, and the value of the quantum yield to be measured. A straightforward analysis of the propagation of errors in the calorimetric method shows that in order to measure a value of $\Phi_{f}=0.5$ with a precision of 1 percent, the heating of the nonfluorescent sample must be measured with a precision of 0.1 percent.

It is shown later that most calorimeters detect the heating power produced by steady state irradiation. If the incident radiation power is $100 \mathrm{~mW}$, and we are using samples that absorb 10 percent of the beam or less to avoid reabsorption effects, then our calorimeter must be capable of measuring a heating power of 10 $\mu \mathrm{W}$ with a signal-to-noise ratio of unity.

It is now obvious that the method for detecting heat flow in the calorimeter must be very sensitive. Most 
calorimeters use temperature transducers to measure heat flow. One figure of merit, useful for evaluating temperature transducers, is their noise equivalent temperature $(N E T)$ given by the following expression

$$
N E T=\left[N / S(\Delta f)^{1 / 2}\right] \operatorname{deg} / \mathrm{Hz}^{1 / 2}
$$

where $N$ is the noise voltage developed by the transducer, $S$ is the voltage sensitivity of the transducer, and $\Delta f$ is the noise bandwidth. The $N E T$ is defined as the temperature change required to generate a signalto-noise ratio of unity when the detector noise is referred to a one cycle bandwidth.

One common method for measuring temperature changes in calorimetry is by means of a thermopile. Generally, the limiting noise in this device is due to the Johnson noise of its resistance. For a 100-junction copper-constantan thermopile of $100 \Omega$ resistance, the $N E T$ is calculated to be $0.2 \mu \mathrm{deg} / \mathrm{Hz}^{1 / 2}$. The tiny voltages associated with these devices can be conveniently measured by means of a commercially available $\mathrm{nV}$ amplifier such as the Keithley Model 148. The construction of thermopiles is an art and is discussed by Hill [109], Howarth [110], and Evans [111].

Resistance thermometers have also been used for calorimetric work. Larsen [112] has shown that the sensitivity of platinum resistance thermometers operated by an ac bridge approaches the theoretical limit imposed by the Johnson noise of the device. He gives a NET of $20.5 \mu \mathrm{deg} / \mathrm{Hz}^{1 / 2}$ for a commercially available $500 \Omega$ platinum resistance thermometer. These tranducers are very stable with time, are smaller in size than thermopiles, and do not require reference junctions.

The use of thermistors in calorimetry has been discussed by Meites et al. [113]. Previously, these temperature transducers have been criticized for calorimetric work because of long term instabilities [112]. Meites et al. found, however, that operation of selected and aged $100 \mathrm{~K}$ thermistors at low power levels $(18 \mu \mathrm{W})$ allowed reliable detection of temperature changes of $10 \mu \mathrm{deg}$ by means of a de bridge and recording potentiometer. The NET as predicted by consideration of the Johnson noise is even lower, $0.7 \mu \mathrm{deg} / \mathrm{Hz}^{1 / 2}$. Attainment of this resolution will probably require use of an ac bridge and phase sensitive detection. Thermistors also offer advantages due to their small sizes and fast response times.

In addition to temperature transducers, one can also employ volume transducers to measure heat flow. The volume change, $\Delta V$, produced at constant pressure in response to a heat change, $\Delta Q$, is given by the formula

$$
\Delta V=\alpha \Delta Q / C_{p} \rho
$$

where $\alpha$ is the thermal expansion coefficient of the sample, $\rho$ is its density, and $C_{p}$ is its heat capacity per gram. A simple dilatometer for measuring volume changes in calorimetric experiments has been used by Seybold, Gouterman, and Callis [29]. In their apparatus, the sample is contained in a cell open to the atmosphere only through a capillary tube. The volume of the solution is visually monitored as it expands up the capillary. Volume changes as small as $7 \times 10^{-5}$ $\mathrm{cm}^{3}$ can be measured; for a $10-\mathrm{cm}^{3}$ ethanol sample this corresponds to a temperature rise of $7 \times 10^{-3}{ }^{\circ} \mathrm{C}$. Further increases in sensitivity in capillary dilatometers can be obtained by using a travelling microscope [114] or a photocell to measure changes in the height of the liquid in the capillary.

A greatly improved technique for measuring volume changes has been developed by Callis, Gouterman, and Danielson [44]. These workers use a capacitor microphone to detect volume changes. They claim a noise equivalent volume $(N E V)$ change of $4 \times 10^{-10} \mathrm{~cm}^{3}$ referred to a $1-\mathrm{Hz}$ bandwidth, which corresponds to a NET of $0.04 \mu \mathrm{deg} / \mathrm{Hz}^{1 / 2}$ for heating $10 \mathrm{~cm}^{3}$ of ethanol. In addition to its sensitivity, this technique has other advantages: (a) The volume change is independent of the size of sample irradiated, whereas the magnitude of the temperature change depends on the size of the sample. (b) Equation (5) is valid even though a uniform temperature is not established if, at all points, $\Delta T$ is small enough so that the equation holds locally. Thus, the sample need not be stirred. (c) The response time of this apparatus is limited only by the transit time for sound in the cell. Disadvantages of the volumetric technique are: (a) The electronics, as originally devised by Callis et al. are ac coupled. Use of a capacitance bridge can overcome this disadvantage, however. (b) The apparatus is sensitive to vibration and acoustical noise and therefore must be isolated from both. (c) For water, an important solvent, $\alpha / \rho C_{p}$ is $\sim 10$ times lower than for organic solvents.

All of the calorimeters we shall discuss in this paper are surrounded by an isothermal shield, and thus all can be characterized by the same energy flow equation

$$
d H / d t=F Y_{h} P(t)-K H(t) .
$$

$H(t)$ is the heat energy retained in the sample, $F$ is the fraction of incident power absorbed by the solute, $P(t)$ is the incident radiation power, and $K$ is the firstorder heat loss constant under the assumption that all heat losses are newtonian. We have neglected heating due to absorption by the solvent and reabsorption of fluorescence photons. These effects are discussed elsewhere [1].

Two cases of eq (6) may be distinguished: (a) When the heat loss term is negligible, the calorimeter is an adiabatic one. (b) When the heat loss term is large, the calorimeter is a conduction calorimeter. When the heating input is exactly balanced by an equal cooling, then the calorimeter is known as a compensation calorimeter.

Let us first consider the use of the conduction type calorimeter as employed by Alentsev [104] for measuring $Y_{h}$. The calorimeter consists of a thin cell suspended in a chamber whose walls are maintained at a constant temperature. Temperature changes are measured by means of a thermopile. The measuring 
junctions are arranged symmetrically around the lateral wall of the cell, and the reference junctions are buried in the isothermal wall. The major source of heat loss is through the thermopile itself. A schematic of the apparatus is shown in figure 6. Since temperature changes are measured, we can use the relationship $H(t)=C_{p}\left[T(t)-T_{0}\right]$ to convert eq (6) to a form involving the instantaneous temperature.

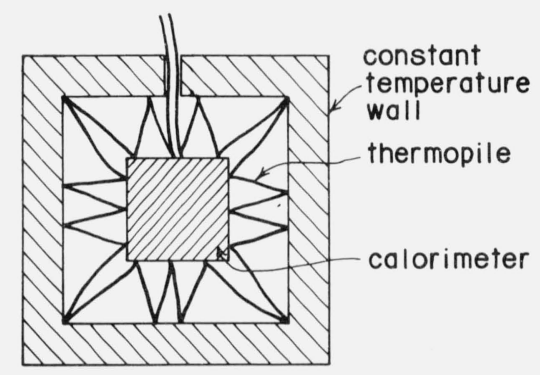

Figure 6. Conduction calorimeter.

$$
d T(t) / d t=\left[F Y_{h} P(t) / C_{p}\right]-K\left[T(t)-T_{0}\right]
$$

where $T_{0}$ is the temperature of the wall. For steady state irradiation $(P$ constant), the solution to eq $(7)$ is

$$
T(t)-T_{0}=F Y_{h} P\left(K C_{p}\right)^{-1}[1-\exp (-K t)] .
$$

After a time $t \gg 1 / K$ the maximum temperature rise, $\Delta T_{m}$, is reached.

$$
\Delta T_{m}=F Y_{h} P\left(K C_{p}\right)^{-1} .
$$

The ratio of $\Delta T_{m}$ for the fluorescent sample to $\Delta T_{m}$ for a nonfluorescent sample that absorbs the same fraction of incident light gives $Y_{h}$, provided $K$ and $C_{p}$ are the same. For a given energy input, $\Delta T_{m}$ can be maximized by using the lowest value of $K$ and $C_{p}$. As used for determination of $Y_{h}$, the conduction calorimeter may be thought of as a power measuring device, and thus a factor of merit is its noise equivalent power $(N E P)$, which is defined as the power input required to generate a signal-to-noise ratio of unity when the detector noise is referred to a $1-\mathrm{Hz}$ bandwidth. For the conduction calorimeter

$$
N E P=N E T(K) C_{p}
$$

where the NET is that of the temperature transducer used. For a NET of $1 \mu \mathrm{deg}$, a heat capacity of 10 $\mathrm{cal} /{ }^{\circ} \mathrm{C}$ and a $K^{-1}$ of $10 \mathrm{~min}$, one obtains a $N E P$ of $0.07 \mu \mathrm{W}$. In practice, the best conduction calorimeters [115] exhibit a $N E P$ of $0.2 \mu \mathrm{W}$; but even this value is far better than that obtained by Alentsev. The limit of sensitivity of this apparatus is apparently determined by the temperature stability of the environment of the calorimeter [115]. Design and construction of conduction type microcalorimeters is discussed extensively by Evans [111] and by Calvet and Prat $[115,116]$. In the most advanced designs $[111,115,116]$, the experiment is conducted differentially, i.e., the temperature difference between the irradiated sample in one cell and an identical unirradiated sample in a twin cell is measured. This technique greatly increases the long term stability of the apparatus and is to be recommended for any type of dc calorimetry.

As employed for conventional calorimetric measurements, conduction calorimeters are not stirred. This is possible if a large number of thermocouples is distributed evenly over the surface of the calorimeter so that the average surface temperature is measured. For fluorescence measurements, however, it is ideal to have all of the fluorescence escape the cell. Thus, the thermopiles should occupy only a tiny fraction of the cell surface. The cell must be stirred. Unfortunately, stirring produces its own heating, which gives rise to additional noise in the system [111].

Perhaps the major disadvantage of using conduction calorimeters is that long times are required for measurement. For a $K^{-1}$ equal to $10 \mathrm{~min}$, one must wait at least $1 \mathrm{~h}$ for the sample to come into equilibrium, and additional time will be taken waiting for the temperature to stabilize.

Some recent work by Chastel and Tachoire [117] suggests that the commercially available and highly perfected Tian-Calvet conduction microcalorimeter may be used in a virtually unmodified form in conjunction with a pulsed light source. The calorimeter is shown schematically in figure 7 . The cell is made of glass or quartz, which is surrounded by a metallic "can" whose inside is coated with a totally absorbing black substance. The thermocouples are

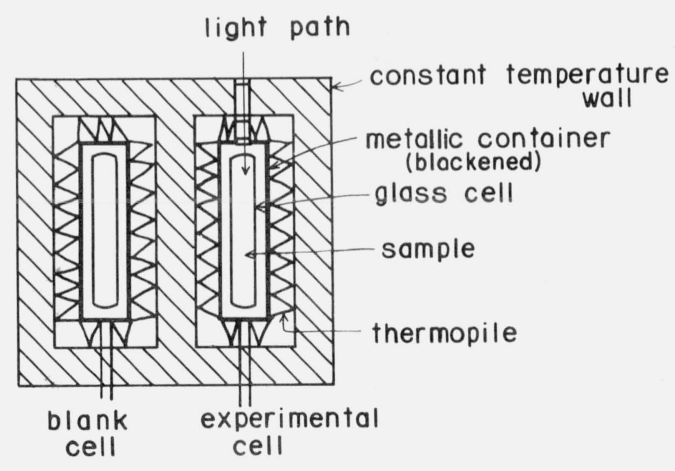

FIGURE 7. Tian-Calvet conduction microcalorimeter modified for quantum-yield determination.

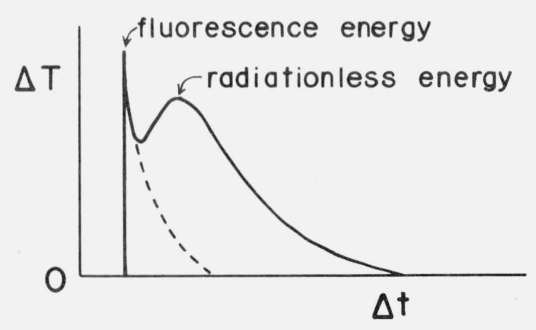

FigURE 8. Thermogram from Tian-Calvet microcalorimeter. 
affixed to the metallic cell. Irradiation of the sample produces a double flux of light and heat. The light is converted to heat through absorption by the metallic cylinder. A recording of the temperature as registered by the thermocouples versus time in the form of a thermogram would be expected to yield the results shown in figure 8. The first peak is due to light absorbed at the cylinder; the second is due to heat diffusing from the sample produced by radiationless processes. If the response times of the apparatus to heat and light are determined, then it is easy to separate the two responses and obtain the energy yields. Chastel and Tachoire have obtained excellent results on energy yields of flash sources for photography in this manner.

A second type of calorimeter, which can be used for measurements of $Y_{h}$, is a heat flow compensation calorimeter. The design of such calorimeters has been discussed by Sturtevant [118]. In these devices, heating produced by irradiation is balanced by an equal amount of cooling power supplied by a thermoelectric module. The null heating point is sensed by a temperature transducer. Thus, the cooling power supplied is a direct measure of the energy input power. An apparatus is shown schematically in figure 9. This device

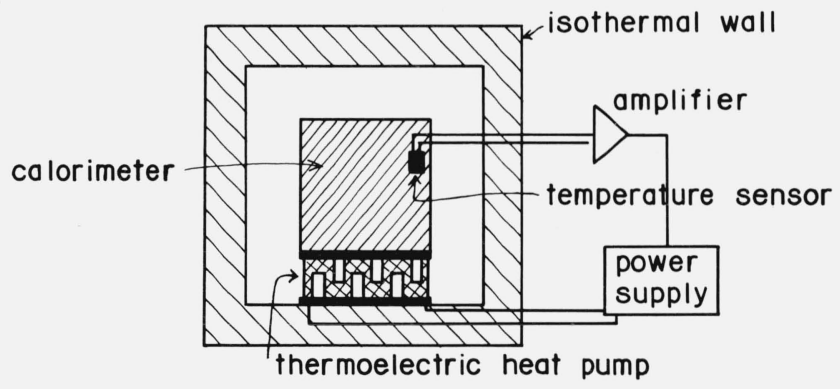

Figure 9. Heat flow compensation calorimeter.

is also essentially a power measuring apparatus, and its factor of merit is given by the $N E P$, which is related to the NET of the temperature sensor, as follows:

$$
N E P=N E T\left(C_{p}\right) \tau^{-1}
$$

where $\tau$ is the time constant of the temperature detection. To minimize the $N E P$, one desires the lowest values of heat capacity and $N E T$ and the longest time constant. For a $N E T=1 \mu \mathrm{deg}, C_{p}=10 \mathrm{cal} /{ }^{\circ} \mathrm{C}$, and $\tau=10$ the $N E P$ is $4.2 \mu \mathrm{W}$. This type of calorimeter may also be used in a differential mode where the difference between an irradiated and unirradiated cell is measured.

Adiabatic calorimeters are a third possibility for $Y_{h}$ measurements. They have been employed in this manner by Seybold, Gouterman, and Callis [29] and by Gudmundson et al. [119]. In these devices one attempts to eliminate heat transfer between the calorimeter and its surroundings. Because of the tiny temperature changes involved, this ideal is best approached by conducting experiments in a constant temperature jacket calorimeter for short time periods so that heat losses are negligible. As with conduction calorimeters, adiabatic calorimeters can be used with steady or pulsed sources. If the sample is irradiated by a steady light source, the temperature rise can be measured as a function of time. In this case, the calorimeter may be thought of as a power measuring device, and the minimum detectable power level will be related to the $N E T$ of the temperature sensor by the formula

$$
N E P=N E T\left(C_{p}\right) \Delta t^{-1}
$$

where $\Delta t$ is the maximum time after the start of irradiation at which a measurement can be made. For the isothermal jacket calorimeter this condition will be $K \Delta t \ll 1$ where $K$ is the rate of newtonian heat loss. For a $N E T$ of $1 \mu \mathrm{deg}$, a $C_{p}$ of $10 \mathrm{cal} /{ }^{\circ} \mathrm{C}$, and a $\Delta t$ of $100 \mathrm{~s}$, a $N E P$ of $0.42 \mu \mathrm{W}$ is obtained. If a pulsed light source is used, the net temperature rise is measured. In this case, the calorimeter determines the integral of the incident power, and thus a figure of merit is the noise equivalent energy change, $N E E$, which is just the NET of the temperature sensor times the heat capacity of sample and calorimeter. Thus, for a $N E T$ of $1 \mu \mathrm{deg}$ and a $10 \mathrm{cal} /{ }^{\circ} \mathrm{C}$ heat capacity, we obtain a $N E E$ of $10 \mu \mathrm{cal}$. This method is attractive because pulsed light sources of high energy are readily available (e.g., pulsed lasers), and heating due to absorption of fluorescence by the calorimeter can be separated by its time dependence.

Thus far, all of the types of calorimeters we have discussed are, in principle, capable of measuring very low power levels and will allow elimination of reabsorption corrections. They all have a common single disadvantage, however. The measurements are performed near zero frequency and are tedious and time consuming. Further, the temperature of the calorimeter and surroundings must be controlled to the NET of the temperature sensing device if the lowest possible $N E P$ of the calorimeter is to be realized.

Currently, a new technique for calorimetric measurements of $Y_{h}$, which can overcome some of the disadvantages of conventional calorimetry, is under development. Essentially the apparatus is the flash calorimeter of Callis, Gouterman, and Danielson [44] shown in figure 10. It consists of a glass cuvette, one wall of which is the compliant diaphragm of a capacitor microphone. The valve attached to one side of the cell serves as a capillary leak so that the liquid can slowly enter or leave the cell. This prevents slow pressure changes that would occur in a completely closed cell subject to temperature drifts and the dc component of repetitive excitation.

Because of the fast response time of this apparatus, the exciting light is modulated, and the resultant heating is detected at the frequency of the modulation by phase sensitive techniques. The advantages of ac methods of doing experiments is well known [120]. Briefly, all experiments are contaminated with noise, some of which has a $1 / f^{n}$ frequency dependence. This latter noise is dominant in calorimetric measure- 

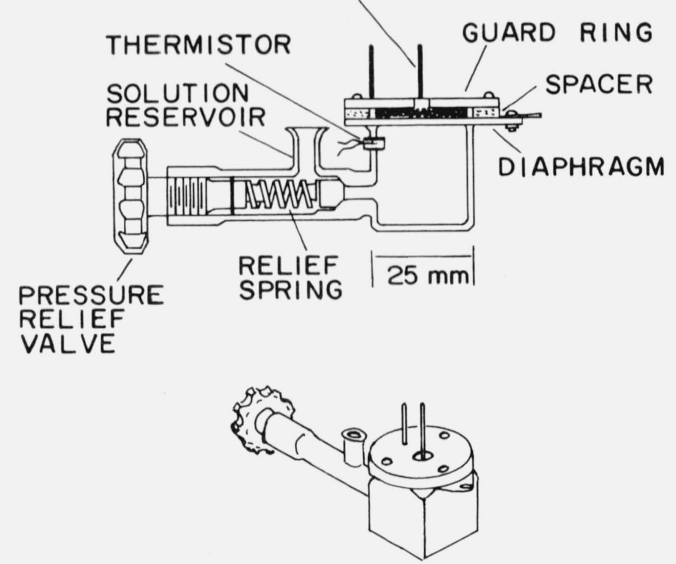

Figure 10. Flash calorimeter.

ments, which are performed at low frequencies where thermal drifts are important. By modulation one can shift the frequency domain of the experiment to a point where noise due to thermal drifts is no longer important and thermostating can be eliminated. By means of phase sensitive detection, very narrow bandwidths can also be obtained, further discriminating against noise.

Due to these advantages, it is worthwhile to consider in more detail this new method for determining $Y_{h}$. Assume that the light intensity has the time dependent form

$$
P(t)=P_{0}(1+\cos \omega t) .
$$

$P_{0}$ is the steady state power in cal/s and $\omega$ is the modulation frequency. The heating in the cell in response to the excitation is given by eq (6). We may combine eqs (5) and (6) to obtain a differential equation for the time dependence of the volume change

$$
d V(t) / d t=\left[\alpha F Y_{h} / \rho C_{p}\right] P(t)-K^{\prime} V(t)
$$

where $K^{\prime}$ is the first-order rate constant for removal of the excess volume change and is given by

$$
K^{\prime}=\alpha K / C_{p}+K^{\prime \prime} .
$$

In this expression, $K^{\prime \prime}$ is the rate constant for the capillary leak. Integration of eq (14) gives for the steady state solution

$$
V(t)=Y_{h} P_{0}(\cos \omega t) / \rho C_{p} \omega
$$

where we have assumed that $\omega \gg K^{\prime}$.

The amplitude of the signal will, therefore, be inversely dependent upon the frequency of modulation. In practice, the experiment should be done at the lowest frequency commensurate with unimportant $1 / f$ noise. This apparatus, like the other calorimeters discussed, is also a power detector, and its $N E P$ will be given by

$$
N E P=N E V\left(C_{p}\right) \rho \omega / \alpha .
$$

For a $N E V$ of $10^{-10} \mathrm{~cm}^{3}, \rho C_{p} / \alpha=4 \times 10^{2} \mathrm{cal} / \mathrm{cm}^{3}$, and $\omega=1 \mathrm{~s}^{-1}$, we obtain a $N E P$ of $0.2 \mu \mathrm{W}$. In addition to its great sensitivity, the advantages of this technique over others we have thus far discussed are the elimination of elaborate thermostating and the execution of measurements rapidly.

An alternative method for using the flash calorimeter is to employ a pulsed exciting source. If a fairly high repetition rate is achieved, the volume change can be stored in a computer and averaged over many repetitions. If the noise is random, then the improvement in signal-to-noise ratio will increase as the square root of the number of experiments averaged.

\section{B. Light Sources}

The ideal light source for calorimetric measurements must be of high intensity, stable with time, and of high spectral purity. Thus far, only conventional noncoherent light sources have been used. Tungsten lamps are very stable with time but give useful output only in the visible region of the spectrum; further, their high infrared output is difficult to remove. High pressure mercury and mercury-xenon arc lamps appear to be the most useful of the noncoherent light sources. They generate high-intensity pressurebroadened lines in the visible and ultraviolet, produce a tolerable infrared output, and can be easily stabilized. Monochromatization of light from noncoherent sources has been achieved through use of solution filters, but complete removal of infrared light is not possible. In contrast, interference filters can be fabricated to specification and will remove most unwanted infrared radiation.

Potentially the most useful light sources for calorimetric yield measurements are lasers, just as they are for optical quantum-yield measurements. As discussed above, there are now commercially available several different types of $\mathrm{cw}$ gas lasers that lase at a variety of frequencies in the spectral region 320 to $650 \mathrm{~nm}$. The $2-\mathrm{W}$ argon ion laser is a good example (see table 1). Other gas phase lasers commercially available and of possible use in calorimetry are $\mathrm{Kr}, \mathrm{He}-\mathrm{Ne}, \mathrm{He}-\mathrm{Cd}$, Se, and Xe. The amplitude stability of these lasers can be made as high as 0.1 percent. Generally, there is some noncoherent plasma radiation in the beam, but this may be removed by interference filters and the use of long pathlengths. The principal drawback to these devices is that their outputs are limited to specific lines, and it is always desirable to excite a molecule at the maximum of its absorption. The availability of the laser-pumped dye lasers should remedy this problem. The narrow line widths, stable outputs, and tuning capabilities over wide spectral regions make them prime candidates for sources in calorimetric quantum-yield determinations. 
If a pulsed light source can be used, then the flashlamp-pumped dye laser is an ideal choice. As discussed previously, these sources can be tuned continuously in the region $340-600 \mathrm{~nm}$ using several different dyes, and their extension farther into the infrared is already at hand. Good short term amplitude stability and high energies per pulse are commercially available in coaxially pumped systems.

\section{Conclusions}

Calorimetric methods for measuring quantum yields have been little used in the past because of the difficulty of making the measurements and, to some extent, because of the average photochemist's unfamiliarity with the techniques. Recent advances in the technology of calorimetry, the progress in transducer design, and the availability of laser excitation sources promise considerable improvements in the rapidity and accuracy of calorimetric methods. These improvements should lead to wider appreciation of this powerful technique for attacking the fundamental problem of measuring radiative and radiationless efficiencies of excited molecular systems.

\section{References}

[1] Demas, J. N., and Crosby, G. A., J. Phys. Chem., 75, 991 (1971).

[2] Lipsett, F. R., Progr. Dielectrics, 7, 217 (1967).

[3] Saxena, V. N., Ind. J. Pure Appl. Phys., 9,407 (1971).

[4] Saxena, V. N., Rense, W. A., and Bruner, E. C., Jr., J. Opt. Soc. Amer., 60, 865 (1970).

[5] Thornton, W. A., J. Electrochem. Soc.,116, 286 (1969).

[6] Bruner, E. C., Jr., J. Opt. Soc. Amer., 59, 204 (1969).

[7] Hammer, A., and Wolf, H. C., Phys. Status Solidi, 33, K25 (1969).

[8] Nygaard, K. J., Brit. J. Appl. Phys., 15,597 (1964).

[9] Kristianpoller, N., J. Opt. Soc. Amer., 54, 1285 (1964).

[10] Allison, R., Burns, J., and Tuzzolino, A. J., ibid., 54, 747 (1964).

[11] Al-Ani, Kh., and Phillips, D., J. Phys. Chem., 75, 3662 (1971).

[12] Borisevich, N. A., and Tolstorozhev, G. B., Bull. Acad. Sci., USSR, Phys. Ser., 34, 574 (1970).

[13] Nakamura, K., J. Chem. Phys., 53, 998 (1970).

[14] Robinson, G. W., and Frosch, R. P., ibid., 3 7, 1962 (1962).

[15] Robinson, G. W., and Frosch, R. P., ibid., 38, 1187 (1963).

[16] Siebrand, W., in The Triplet State, A. B. Zahlan, Ed. (Cambridge University Press, London, 1967), p. 31.

[17] Siebrand, W., J. Chem. Phys., 47, 2411 (1967).

[18] Siebrand, W., ibid., 46, 440 (1967).

[19] Lin, S. H., ibid., 44, 3759 (1966).

[20] Bixon, M., and Jortner, J., ibid., 48, 715 (1968).

[21] Henry, B. R., and Siebrand, W., ibid., 54, 1072 (1971).

[22] Burland, D. M., and Robinson, G. W., ibid., 51, 4548 (1969).

[23] Rusakowicz, R., and Testa, A. C., Spectrochim. Acta, 27A, 787 (1971).

[24] Klueger, J., Fischer, G., Fischer, E., Goedicke, Ch., and Stegemeyer, H., Chem. Phys. Lett., 8, 279 (1971).

[25] Lentz, P., Blume, H., and Schulte-Frohlinde, D., Ber. Bunsenges. Phys. Chem., 74, 484 (1970).

[26] Sharafy, S., and Muszkat, K. A., J. Amer. Chem. Soc., 93, 4119 (1971).

[27] Eastman, J. W., Spectrochim. Acta., 26A, 1545 (1970).

[28] Strickler, S. J., and Berg, R. A., J. Chem. Phys., 3 7, 814 (1962).

[29] Seybold, P. G., Gouterman, M., and Callis, J., Photochem. Photobiol., 9, 229 (1969).

[30] Douglas, A. E., J. Chem. Phys., 45, 1007 (1966).

[31] Birks, J. B., and Dyson, D. J., Proc. Roy. Soc., Ser. A, 275, 135 (1963).

[32] Briegleb, G., Trencseni, J., and Herre, W., Chem. Phys. Lett., 3, 146 (1969).
[33] Kobayashi, T., Yoshihara, K., and Nagakura, S., Bull. Chem. Soc. Japan, 44, 2603 (1971).

[34] El-Bayoumi, M. A., Dalle, J. D., and O'Dwyer, M. F., J. Amer. Chem. Soc., 92, 3494 (1970).

[35] Demas, J. N., and Crosby, G. A., ibid., 92, 7262 (1970).

[36] Demas, J. N., and Crosby, G. A., ibid., 93, 2841 (1971).

[37] Watts, R. J., and Crosby, G. A., ibid., 94, 2606 (1972).

[38] Harrigan, R. W., and Crosby, G. A., Abstracts, 163rd National Meeting of the American Chemical Society, Boston, Mass., April 1972, Phys. 105.

[39] Hager, G. D., and Crosby, G. A., 1972, unpublished results.

[40] Englman, R., and Jortner, J., Mol. Phys., 18, 145 (1970).

[41] Lim, E. C., and Bhattacharjee, H. R., Chem. Phys. Lett., 9, 249, (1971).

[42] Ricci, R. W., Photochem. Photobiol., 12,67 (1970).

[43] Thomas, T. R., Watts, R. J., and Crosby, G. A., J. Chem. Phys. (in press).

[44] Callis, J. B., Gouterman, M., and Danielson, J. D. S., Rev. Sci. Instrum., 40, 1599 (1969).

[45] Bonnier, J. M., and Jardon, P., J. Chim. Phys. Physicochim. Biol., 67, 577 (1970); 66, 1506 (1969).

[46] Bonnier, J. M., Jardon, P., and Blanchi, J. P., Bull. Soc. Chim. Fr., 12, 4787 (1968).

[47] Wilkinson, F., and Dubois, J. T., J. Chem. Phys., 39, 377 (1963).

[48] Förster, Th., Ann. Physik, 2, 55 (1948).

[49] Weber, G., and Teale, F. W. J., Trans. Faraday Soc., 53, 646 (1957).

[50] Pringsheim, P., Fluorescence and Phosphorescence (Interscience Publishers, Inc., New York, N.Y., 1949).

[51] Bowers, P. G., and Porter, G., Proc. Roy. Soc., Ser. A, 299, 348 (1967).

[52] Medinger, T., and Wilkinson, F., Trans. Faraday Soc., 61, 620 (1965).

[53] Horrocks, A. R., Medinger, T., and Wilkinson, F., Chem. Commun., 452 (1965).

[54] Medinger, T., and Wilkinson, F., Trans. Faraday Soc., 62, 1785 (1966).

[55] Horrocks, A. R., Kearvell, A., Tickle, K., and Wilkinson, F., ibid., 62, 3393 (1966).

[56] Horrocks, A. R., Medinger, T., and Wilkinson, F., Photochem. Photobiol., 6, 21, (1967).

[57] Horrocks, A. R., and Wilkinson, F., Proc. Roy, Soc., Ser. A, 306, 257 (1968).

[58] Gradyushko, A. T., Sevchenko, A. N., Solovyov, K. N., and Tsvirko, M. P., Photochem. Photobiol., 11, 387 (1970).

[59] Ermolaev, V. L., and Sveshnikova, E. B., Acta Phys. Polon., 34, 771 (1968).

[60] Seybold, P. G., and Gouterman, M., J. Mol. Spectrosc., 31, 1 (1969).

[61] Kropp, J. L., Dawson, W. R., and Windsor, M. W., J. Phys. Chem., 73, 1747 (1969).

[62] Gill, J. E., Photochem. Photobiol., 11, 259 (1970).

[63] Leng, M., Pochon, F., and Michelson, A. M., Biochim. Biophys. Acta, 169, 338 (1968).

[64] Pochon, F., Leng, M., and Michelson, A. M., ibid., 169, 350 (1968).

[65] Ward, D. C., Reich, E., and Stryer, L., J. Biol. Chem., 244, 1228 (1969).

[66] Edelman, G. M., and McClure, W. O., Accounts Chem. Res., 1, 65 (1968).

[67] Stryer, L., Science, 162, 526 (1968).

[68] Winkler, M., J. Mol. Biol., 4, 118 (1962).

[69] Kingdon, H., Fed. Proc., 26, 842 (1967).

[70] McClure, W. O., and Edelman, G. M., Biochemistry, 6, 567 (1967).

[71] Crosby, G. A., Harrigan, R. W., and Watts, R. J., patent applied for.

[72] Demas, J. N., and Adamson, A. W., J. Amer. Chem. Soc., 93, 1800 (1971).

[73] Watts, R. J., and Crosby, G. A., ibid., 93, 3184 (1971).

[74] Testa, A. C., Fluorescence News, 4, No. 4, 1 (1969).

[75] Gill, J. E., Photochem. Photobiol., 9, 313 (1969).

[76] Spencer, R. D., Vaughan, W. M., and Weber, G., in Molecular Luminescence, E. C. Lim, Ed. (W. A. Benjamin, New York, N.Y., 1969), p. 607. 
[77] Ness, S., and Hercules, D. M., Anal. Chem., 41, 1467 (1969).

[78] Druding, L. F., and Kauffman, G. B., Coordin. Chem. Rev., 3, 409 (1968).

[79] Clifford, H. J., Ph. D. dissertation, University of New Mexico, 1971.

[80] Vaviloy, S. I., Z. Phys., 22, 266 (1924).

[81] Bowen, E. J., and Sawtell, J. W., Trans. Faraday Soc., 33, 1425 (1937).

[82] Förster, Th., Fluoreszenz Organischer Verbindungen (Vandenhoeck-Ruprecht, Göttingen, Germany, 1951).

[83] Gilmore, E. H., Gibson, G. E., and McClure, D. S., J. Chem. Phys., 20, 829 (1952); 23, 399 (1955).

[84] Melhuish, W. H., New Zealand J. Sci. Tech., 37, 142 (1955).

[85] Almgren, M., Photochem. Photobiol., 8, 231 (1968).

[86] S.P.I.E. Seminar Proceedings, Developments in Laser Technology, Vol. 20, University of Rochester, 1969.

[87] Hanna, D. C., and Smith, R. C., Sci. J., 5, 53 (1969).

[88] Goldstein, A., and Dacol, F. H., Rev. Sci. Instrum., 40, 1597 (1969).

[89] Furumoto, H. W., and Ceccon, H. L., IEEE, J. Quant. Electron., 6, $262(1970)$.

[90] Soffer, B. H., and Linn, J. W., J. Appl. Phys., 39, 5859 (1968).

[91] Snavely, B. B., Proc. IEEE, 5 7, 1374 (1969).

[92] DeMaria, A. J., Glenn, W. H., and Mack, M. E., Phys. Today, 24, 19 (1971).

[93] Sorokin, P., Sci. Amer., 220, 30 (1969).

[94] Smith, R. G., Anal. Chem., 41, 75A (1969).

[95] Tansley, T. L., and Ralph, J. E., J. Phys. D, 3, 807 (1970).

[96] Mathur, D. P., McIntyre, R. J., and Webb, P. P., Appl. Opt., 9, 1842 (1970).

[97] Haecker, W., Groezinger, O., and Pilkuhn, M. H., Appl. Phys. Lett., 19, 113 (1971).

[98] Melhuish, W. H., J. Phys. E, 4, 60 (1971).

[99] Bojarski, C., Kusba, J., and Obermüller, G., Z. Naturforsch., A26, 255 (1971).

[100] Weller, A., and Zachariasse, K., Chem. Phys. Lett., 10, 424 (1971).

[101] Wegner, E. E., and Adamson, A. W., J. Amer. Chem. Soc., 88, 394 (1966).

[102] Demas, J. N., and Adamson, A. W., Manuscript in preparation.
[103] The term fluorescence is used here in the general sense, i.e., to include all radiative processes whether spin allowed or spin forbidden.

[104] Alentsev, M. A., National Research Council of Canad: Technical Translation TT-433, Zh. Eksp. Teor. Fiz., 21, 133 (1951).

[105] Brown, H. D., Ed., Biochemical Microcalorimetry (Academic Press, Inc., New York, N.Y., 1969).

[106] Wadsö, I., Quart. Rev. Biophys., 3, 383 (1970).

[107] McCullough, J. P., and Scott, D. W., Ed., Experimental Thermodynamics, Vol. 1 (Plenum Press, Inc., New York, N. Y., 1968).

[108] Weissberger, A., Ed., Techniques of Organic Chemistry, 3d ed, Vol. 1 (Interscience Publishers, Inc., New York, N.Y., 1959), Ch. 10.

[109] Hill, A. V., Trails and Trials in Physiology (Edward Arnold, London, 1965).

[110] Howarth, J. V., Quart. Rev. Biophys., 3, 429 (1970).

[111] Evans, W. J., in Biochemical Microcalorimetry, H. D. Brown, Ed. (Academic Press, Inc., New York, N.Y., 1969), Ch. 14.

[112] Larsen, N. T., Rev. Sci. Instrum., 39, 1 (1968).

[113] Meites, T., Meites, L., and Jaitly, J. N., J. Phys. Chem., 73, 3801 (1969).

[114] Zamyatnin, A. A., Russ. J. Phys. Chem., 45, 567 (1971).

[115] Calvet, E., and Prat, H., Recent Progress in Microcalorimetry (translated by H. A. Skinner)(The Macmillan Co., New York, N.Y., 1963).

[116] Calvet, E., and Prat, H., Microcalorimetrie (Masson, Paris, 1956).

[117] Chastel, R., and Tachoire, H., Rev. Gen. Elec., 77, 871 (1968).

[118] Buzzell, A., and Sturtevant, J. M., J. Amer. Chem. Soc., 73, 2454 (1951).

[119] Gudmundsen, R. A., Marsh, O. J., and Matovich, E., J. Chem. Phys., 39, 272 (1963).

[120] Wilson, E. B., Introduction to Scientific Research (McGrawHill Book Co., Inc., New York, N.Y., 1952).

(Paper 76A6-742) 